\title{
Some Two-Cardinal Results for O-Minimal Theories
}

\author{
Timothy Bays
}

\begin{abstract}
We examine two-cardinal problems for the class of O-minimal theories. We prove that an O-minimal theory which admits some $(\kappa, \lambda)$ must admit every $\left(\kappa^{\prime}, \lambda^{\prime}\right)$. We also prove that every "reasonable" variant of Chang's Conjecture is true for O-minimal structures. Finally, we generalize these results from the two-cardinal case to the $\delta$-cardinal case for arbitrary ordinals $\delta$.
\end{abstract}

\section{Introduction}

In their most general form, two-cardinal problems depend heavily on assumptions about our background set theory. Admitting cardinals conjectures, for instance, are often true in $L$ but false in relatively straightforward extensions of $L$. Similarly, most variants of Chang's Conjecture have the consistency strength of quite large cardinals.

If we restrict ourselves to stable theories, two-cardinal problems become both more tractable and more amenable to model-theoretic, as opposed to set-theoretic, investigation. Lachlan has shown that any stable theory which admits some $(\kappa, \lambda)$ must admit every $\left(\kappa^{\prime}, \lambda^{\prime}\right)$ (see [4]). Similarly, Shelah has shown that most variants of Chang's Conjecture are true for superstable theories, and the present author has shown that this result generalizes to (almost all) $|T|^{+}$-saturated models of stable theories (see [6] and [2] respectively).

Two features of stable theories make these results possible. First, stable theories have a nice notion of independence (non-forking) which "explains" differences in cardinality between separate parts of a model. Second, stable theories admit an assortment of "prime model" constructions which allow us to build models respecting this independence.

Both of these features are present in O-minimal theories as well (in many ways, O-minimal theories are just $\omega$-stable theories, but without the stability). Like stable theories, O-minimal theories have a nice notion of independence (simple algebraic independence). Like $\omega$-stable theories, O-minimal theories admit prime models over arbitrary sets (see [5]).

In the present paper, we show that O-minimal theories are well-behaved with respect to two-cardinal problems. We begin by proving an O-minimal analog of Lachlan's theorem from [4]. We prove that if $T$ is O-minimal and $P(N) \subset M \prec N$, then there exists $N^{\prime}$ such that $P\left(N^{\prime}\right) \subset M \prec N \prec N^{\prime}$. From this, we conclude that an O-minimal theory which admits some $(\kappa, \lambda)$ must admit every $\left(\kappa^{\prime}, \lambda^{\prime}\right)$.

On the Chang's Conjecture side, we prove that if $M$ is an O-minimal structure of type $(\kappa, \lambda)$ and if $\kappa^{\prime}$ and $\lambda^{\prime}$ are such that $\omega \leq \lambda^{\prime} \leq \kappa^{\prime}, \lambda^{\prime} \leq \lambda$, and $\kappa^{\prime} \leq \kappa$, then there exists $N \prec M$ such that $N$ is of type 
$\left(\kappa^{\prime}, \lambda^{\prime}\right)$. In section 4 , we generalize these results from the two-cardinal case to the $\delta$ cardinal case, where $\delta$ is some arbitrary ordinal.

Throughout the paper, $T$ is countable and $\mathrm{O}$-minimal and $\mathbb{M}$ is a monster model for $T$. We assume basic facts about O-minimal theories. These can be found in [5] and [3]. We always let "<" pick out the order of $\mathbb{M}$, and we assume that the "small" portions of our two-cardinal models are picked out by the predicate "P". Using the fact that $T$ is O-minimal, we fix $\hat{c}_{1}, \ldots, \hat{c}_{2 n} \in \mathbb{M} \cup\{ \pm \infty\}$ and $\hat{d}_{1}, \ldots, \hat{d}_{m} \in \mathbb{M}$ such that $P(\mathbb{M})=\bigcup_{i=1}^{n}\left\{x \mid \hat{c}_{1}<x<\hat{c}_{i+1}\right\} \cup \bigcup_{i=1}^{m}\left\{\hat{d}_{i}\right\}$.

Notationally, we use $M, N, \ldots$ to denote models and $A, B, \ldots$ to denote subsets of models. We use $\alpha, \beta, \gamma, \ldots$ to denote ordinals; $\kappa, \lambda, \mu, \ldots$ to denote infinite cardinals; $m$ and $n$ to denote natural numbers; and $i, j, k$ and $l$ to denote either ordinals or natural numbers depending on the context. We use "२" to mean $\supsetneqq$.

\section{Admitting Cardinals}

The next lemma is, in some sense, the key to the entire paper. It shows that prime model constructions respect algebraic independence vis-a-vis definable subsets of $\mathbb{M}$.

Lemma 1 Let $M$ be a model and let $A \supset M$ be algebraically closed and such that $P(A) \subset M$. Let $M[A]$ be prime over $A$. Then $P(M[A]) \subset M$.

Proof. Suppose not, and let $b \in P(M[A]) \backslash M$. As $M[A]$ is atomic over $A$, there is some formula $\psi(x, \bar{a})$ over $A$ such that $\psi(x, \bar{a})$ isolates $\operatorname{tp}(b, A)$. Since $A$ is algebraically closed, we may assume that $\psi$ is of the form " $a_{1}<x<a_{2}$ " where $a_{1}, a_{2} \in A \cup\{ \pm \infty\}$. Further, since $\psi$ both isolates $\operatorname{tp}(b, A)$ and entails that $b$ is in $P(\mathbb{M})$, there must be some $i$ such that $\psi(x) \vdash \hat{c}_{i}<x<\hat{c}_{i+1}$. Hence, $\hat{c}_{i} \leq a_{1}<a_{2} \leq \hat{c}_{i+1}$.

Because of this, and because $P(A) \subset M$, both $a_{1}$ and $a_{2}$ must live in $M$. Thus, since $a_{1}<b<a_{2}$ and $M \prec \mathbb{M}, M$ must satisfy " $\exists x\left(a_{1}<x<a_{2}\right)$ ". Hence, for some $n_{1} \in M \cap\left(a_{1}, a_{2}\right), \psi$ does not decide either " $a_{1}<x<n_{1}$ " or " $n_{1}<x<a_{2}$ ". So, $\psi$ fails to isolate $\operatorname{tp}(b, A)$ for a contradiction.

Lemma 2 Let $P(N) \subset M \prec N$ and let $a \in N \backslash M$. Let $A \supset N$ be algebraically closed, let $p \in S(A)$ be an heir of $t p(a, M)$, and let $b \models p$. Then, $P(a c l(A b)) \subset A$.

Proof. Suppose not, and let $c \in P(\operatorname{acl}(A b)) \backslash A$. Since $c \in \operatorname{acl}(A b)$ and $c \notin A, b \in \operatorname{acl}(A c)$. Let $\psi(x, \bar{m}, \bar{a}, c)$ witness this. Then,

$$
\models \exists y\left[P(y) \wedge \exists^{=n} x \psi(x, \bar{m}, \bar{a}, y) \wedge \psi(b, \bar{a}, \bar{n}, y)\right] .
$$

Since $\operatorname{tp}(b, A)$ is an heir of $\operatorname{tp}(a, M)$, there is some $\bar{m}^{\prime}$ such that,

$$
\vDash \exists y\left[P(y) \wedge \exists^{=n} x \psi\left(x, \bar{m}, \bar{m}^{\prime}, y\right) \wedge \psi\left(a, \bar{m}, \bar{m}^{\prime}, y\right)\right] .
$$

Since $N$ must satisfy this formula, and since $P(N) \subset M$, we conclude that $a$ must be algebraic over $M$. But, this contradicts $a \in N \backslash M$. 
Theorem 3 Suppose $M \prec N$ and $P(N)=P(M)$. Then there exists $N^{\prime}$ such that $N \prec N^{\prime}$ and $P\left(N^{\prime}\right)=$ $P(M)$.

Proof. Choose $a \in N \backslash M$, and let $p \in S(N)$ be an heir of $\operatorname{tp}(a, M)$. Let $b \models p$. Then by lemma 2, $P(\operatorname{acl}(N b)) \subset N$. So, $P(\operatorname{acl}(N b)) \subset M$. Let $N^{\prime}$ be prime over acl $(N b)$. By lemma $1, P\left(N^{\prime}\right) \subset M$ as desired.

Theorem 4 If $T$ admits some $(\kappa, \lambda)$ where $\kappa>\lambda$, then $T$ admits every $\left(\kappa^{\prime}, \lambda^{\prime}\right)$.

Proof. Let $M$ witness the fact that $T$ admits $(\kappa, \lambda)$. By the downward Löwenheim-Skolem theorem, there exists $M^{\prime} \prec M$ such that $P(M) \subset M^{\prime}$. Let " $U$ " be a fresh predicate and expand $M$ by letting $U(M)=M^{\prime}$. By compactness, we can obtain a model $N$ such that $N \models \operatorname{Th}(\langle M, U\rangle)$ and $|N|=|U(N)|=\lambda^{\prime}$. Note that since $N$ satisfies $\operatorname{Th}(\langle M, U\rangle), P(N) \subset U(N)$ and, vis-a-vis our original language, $U(N) \prec N$.

Returning to our original language, we let $N^{\prime}$ be the submodel of $N$ which was picked out by " $U$ ". By induction, we construct a strictly increasing sequence of models, $\left\langle N_{i} \mid i \leq \kappa^{\prime}\right\rangle$, such that for every $i \leq \kappa^{\prime}$, $P\left(N_{i}\right)=P\left(N^{\prime}\right)$. We start by letting $N_{0}=N$. Given $N_{i}$, we apply theorem 3 to obtain $N_{i+1}$. Finally, for limit $i$, we let $N_{i}=\bigcup_{j<i} N_{j}$. At the end of the day, $N_{\kappa^{\prime}}$ is a $\left(\kappa^{\prime}, \lambda^{\prime}\right)$-model as desired.

Remarks: (1.) Lemma 2 resembles a result from stability theory. In stability theory, we say that a type $p \in S(B)$ is foreign to some definable set $P$ if for every $A \supset B$ and every $p^{\prime} \in S(A)$ a non-forking extension of $p$, if $b \models p^{\prime}$ and $\mathbb{M} \models P(c)$, then $b \downarrow_{A} c$. For stable theories, if $P(N) \subset M \prec N$ and $a \in N \backslash M$, then $\operatorname{tp}(a, M)$ is foreign to $P$ (see [2]). Lemma 2 says the same thing, modulo the need to redefine "non-forking extension" and " $b \downarrow_{A} c$ " so as to make sense in the O-minimal context.

(2.) Note that the proof of theorem 3 actually gives something slightly stronger than theorem 3 itself. Let $M$ and $N$ be as in the theorem and let $N^{\prime} \supset N$ (here, $P\left(N^{\prime}\right)$ need not be a subset of $M$ ). Then our proof shows that there must exist $N^{\prime \prime}$ such that $N^{\prime} \prec N^{\prime \prime}$ and $P\left(N^{\prime \prime}\right)=P\left(N^{\prime}\right)$. So, any model which contains a pair of models like those in theorem 3, can be extended without adding new members of $P(\mathbb{M})$. This will be important in section 4.

(3.) We have proved theorem 4 for countable languages only (since countability is a background assumption for this paper). The result extends trivially to uncountable languages, however. If an uncountable theory $T$ admits some $(\kappa, \lambda)$ (where $\kappa>\lambda)$, then all its countable subtheories admit every $\left(\kappa^{\prime}, \lambda^{\prime}\right)$ (by theorem 4 ). Hence, by a result of Vaught's, $T$ itself admits every $\left(\kappa^{\prime}, \lambda^{\prime}\right)$ (see [7]).

\section{Chang's Conjectures}

Our first lemma "relativizes" theorem 3 so as to work within a particular two-cardinal model. Given a twocardinal model $M$, the lemma allows us to expand arbitrary submodels of $M$, without adding new members of $P(M)$ to these submodels. 
Lemma 5 Let $M$ be a model of type $(\kappa, \lambda)$ where $\kappa>\lambda$. Let $N \prec M$ be such that $|N|<\kappa$. Then there exists $N^{\prime}$ such that $N \prec N^{\prime} \prec M,\left|N^{\prime}\right|=|N|$, and $P\left(N^{\prime}\right)=P(N)$.

Proof. Let $M$ and $N$ be as given. We define an equivalence relation on $M \backslash N$ by,

$$
m_{1} \sim_{N} m_{2} \Longleftrightarrow m_{1} \in \operatorname{acl}\left(N m_{2}\right) \Longleftrightarrow m_{2} \in \operatorname{acl}\left(N m_{1}\right)
$$

Since each equivalence class has cardinality at most $|N|$, this relation partitions $M \backslash N$ into $\kappa$ different pieces. Since there are only $\lambda$ members of $P(M)$, some equivalence class does not contain members of $P(M)$.

Let $A$ be some such equivalence class. Then $N \cup A$ is algebraically closed, and $P(N \cup A) \subset N$. Let $N[A] \prec M$ be prime over $N \cup A$. Clearly, $|N[A]|=|N|$, and by lemma $1, P(N[A])=P(N)$. Hence, $N[A]$ is the desired $N^{\prime}$.

Theorem 6 Let $M$ be a model of type $(\kappa, \lambda)$ where $\kappa>\lambda$. Suppose that $\lambda^{\prime} \leq \lambda, \kappa^{\prime} \leq \kappa$, and $\omega \leq \lambda^{\prime} \leq \kappa^{\prime}$. Then there exists $N \prec M$ such that $N$ is of type $\left(\kappa^{\prime}, \lambda^{\prime}\right)$.

Proof. Using the downward Löwenheim-Skolem theorem, we construct $M^{\prime} \prec M$ such that $\left|M^{\prime}\right|=\left|P\left(M^{\prime}\right)\right|=$ $\lambda^{\prime}$. By induction, we construct a strictly increasing sequence of models, $\left\langle N_{i} \mid i \leq \kappa^{\prime}\right\rangle$, such that for every $i \leq \kappa^{\prime}, N_{i} \prec M, P\left(N_{i}\right)=P\left(M^{\prime}\right)$, and $\left|N_{i}\right|=\lambda^{\prime}+|i|$.

We begin by letting $N_{0}=M^{\prime}$. Given $N_{i}$ where $i<\kappa^{\prime}$, we apply lemma 5 to obtain $N_{i+1}$. Finally, for limit $i$, we let $N_{i}=\bigcup_{j<i} N_{j}$. At the end of the day, $\left|N_{\kappa^{\prime}}\right|=\kappa^{\prime}$ and $\left|P\left(N_{\kappa^{\prime}}\right)\right|=\left|P\left(M^{\prime}\right)\right|=\lambda^{\prime}$ as desired.

Remark: Note that this proof of theorem 6 allows us a great deal of freedom in choosing the "bottom" portion of our $\left(\kappa^{\prime}, \lambda^{\prime}\right)$-models. In particular, for any $M^{\prime} \prec M$, there exist arbitrarily large $N$ such that $M^{\prime} \prec N \prec M$ and $P(N)=P\left(M^{\prime}\right)$.

\section{$4 \delta$-Cardinal Theorems}

In this section we generalize theorems 4 and 6 to the $\delta$-cardinal case. For notational convenience, we choose a fixed sequence of formulas over $\mathbb{M},\left\langle\psi_{i}\left(x, \bar{m}_{i}\right) \mid i<\delta\right\rangle$, and we let $A \subset \mathbb{M}$ be minimal such that every $\psi_{i}$ is also over $A$.

Theorem 7 Suppose that for every $i<\delta$, there exists $M_{i}$ such that $A \subset M_{i}$ and $\left|\psi_{i}\left(M_{i}\right)\right|>\left|\bigcup_{j<i} \psi_{j}\left(M_{i}\right)\right|$. Let $F: \delta \rightarrow C A R D$ be increasing and such that $F(0) \geq|\delta|+\omega$. Then there exists a model $M_{F}$ such that $A \subset M_{F}$ and for every $i<\delta,\left|\psi_{i}\left(M_{F}\right)\right|=F(i)$.

Proof. We construct by induction a strictly increasing sequence of models, $\left\langle N_{i} \mid i<\delta\right\rangle$, such that:

1. for every $i, A \subset N_{i}$,

2. for every $i,\left|\psi_{i}\left(N_{i}\right)\right|=\left|N_{i}\right|=F(i)$, and

3. for every $j<i<\delta, \psi_{j}\left(N_{i}\right)=\psi_{j}\left(N_{j}\right)$. 
At the end of the day, $N_{\delta}=\bigcup_{i<\delta} N_{i}$ will be the desired $M_{F}$.

For $i=0$, we argue as in the proof of theorem 4. Using compactness, we obtain a sequence of pairs, $\left\langle M_{i}^{\prime \prime} \prec M_{i}^{\prime} \mid i<\delta\right\rangle$, such that for every $i, j<\delta$,

- $A \cup \bigcup_{j<i} \psi_{j}\left(M_{i}^{\prime}\right) \subset M_{i}^{\prime \prime}$

- $\psi_{i}\left(M_{i}^{\prime}\right) \not \subset M_{i}^{\prime \prime}$, and

- $\left|\psi_{j}\left(M_{i}^{\prime}\right)\right|=\left|M_{i}^{\prime}\right|=F(0)$.

We then let $N_{0}$ be arbitrary such that $\left|N_{0}\right|=F(0)$ and $\bigcup_{i<\delta} M_{i}^{\prime} \subset N_{0}$.

For $i>0$, we assume that $N_{j}$ has been defined for all $j<i$. By induction, we construct a strictly increasing sequence, $\left\langle N_{k}^{i} \mid k<F(i)\right\rangle$, such that for every $k<F(i)$ and $j<i, \psi_{j}\left(N_{k}^{i}\right)=\psi_{j}\left(N_{j}\right)$. We start by letting $N_{0}^{i}=\bigcup_{j<i} N_{j}$. Note that by our induction hypothesis on the $N_{j}$ 's (clause 3 , in particular), $\psi_{j}\left(N_{0}^{i}\right)=\psi_{j}\left(N_{j}\right)$ as desired. Similarly, for $k$ limit, we simply let $N_{k}^{i}=\bigcup_{l<k} N_{l}^{i}$.

For $k$ a successor, we we choose $a \in \psi_{i}\left(M_{i}^{\prime}\right) \backslash M_{i}^{\prime \prime}$, we let $p \in S\left(N_{k-1}^{i}\right)$ be an heir of $\operatorname{tp}\left(a, M^{\prime \prime}\right)$, and we let $b=p$. By lemma $2, \psi_{j}\left(\operatorname{acl}\left(b N_{k-1}^{i}\right)\right) \subset N_{k-1}^{i}$ for every $j<i$. We let $N_{k}^{i}$ be prime over acl $\left(b N_{k-1}^{i}\right)$. Then by lemma $1, \psi_{j}\left(N_{k}^{i}\right) \subset N_{k-1}^{i}$ for every $j<i$. So, by the induction hypothesis on $N_{k-1}^{i}, \psi_{j}\left(N_{k}^{i}\right)=\psi_{j}\left(N_{j}\right)$ as desired.

When the construction of $\left\langle N_{k}^{i} \mid k<F(i)\right\rangle$ is finished, we set $N_{i}=\bigcup_{k<F(i)} N_{k}^{i}$. Note that conditions 1 and 2 on our induction are satisfied trivially (as $A \subset N_{0} \subset N_{i}$, and the $N_{k}^{i}$-construction added exactly $F(i)$ pieces of $\psi_{i}(\mathbb{M})$ to $\left.N_{i}\right)$. Condition 3 is satisfied because of the induction hypothesis on the subsidiary $N_{k}^{i}$-construction.

Remark: Note that theorem 7 does not require us to start with an initial $\delta$-cardinal model which witnesses all of the relevant cardinality splits simultaneously. We can start with a collection of models, each witnessing a different cardinality split, and then parlay these models into a single $\delta$-cardinal model which exhibits some desired sequence of cardinality splits.

Theorem 8 Let $M \supset A$ be such that for every $i<\delta,\left|\psi_{i}(M)\right|>\left|\bigcup_{j<i} \psi_{j}(M)\right|$. Let $F: \delta \rightarrow C A R D$ be increasing and such that for every $i<\delta, \delta+\omega \leq F(i) \leq\left|\psi_{i}(M)\right|$. Then there exists $N_{F} \prec M$ such that $A \subset N_{F}$ and for every $i<\delta,\left|\psi_{i}\left(N_{F}\right)\right|=F(i)$.

Proof. We construct by induction a strictly increasing sequence of models $\left\langle N_{i} \mid i<\delta\right\rangle$, such that:

1. for every $i, A \subset N_{i} \prec M$,

2. for every $i,\left|\psi_{i}\left(N_{i}\right)\right|=\left|N_{i}\right|=F(i)$, and

3. for every $j<i<\delta, \psi_{j}\left(N_{i}\right)=\psi_{j}\left(N_{j}\right)$.

At the end of the day, we simply let $N_{F}=\bigcup_{i<\delta} N_{i}$. 
For $i=0$, we apply the downward Löwenheim-Skolem theorem to obtain $N_{0} \prec M$ such that $A \subset N_{0}$ and for every $i<\delta,\left|\psi_{i}\left(N_{0}\right)\right|=\left|N_{0}\right|=F(0)$. For $i>0$, we assume that $N_{j}$ has been defined for every $j<i$, and we let $\hat{N}_{i}=\bigcup_{j<i} N_{j}$. Note that for $j<i, \psi_{j}\left(\hat{N}_{i}\right)=\psi_{j}\left(N_{j}\right)$. Next, we let $B_{i} \subset \psi_{i}(M)$ be maximal such that $\hat{N}_{i} \cup B_{i}$ is algebraically closed and for every $j<i, \psi_{j}\left(\hat{N} \cup B_{i}\right) \subset \hat{N}_{i}$.

Claim: $\left|B_{i}\right|=\left|\psi_{i}(M)\right|$

Pf. Suppose $\left|B_{i}\right|<\left|\psi_{i}(M)\right|$. As in the proof of theorem 5, we partition $\psi_{i}(M) \backslash B_{i}$ via the equivalence relation: $a \sim b \Longleftrightarrow a \in \operatorname{acl}\left(\hat{N}_{i} \cup B_{i} \cup\{b\}\right)$. Since there are $\left|\psi_{i}(M)\right|$ equivalence classes and $<\left|\psi_{i}(M)\right|$ elements in $\left|\bigcup_{j<i} \psi_{j}(M)\right|$, some equivalence class does not intersect $\left|\bigcup_{j<i} \psi_{j}(M)\right|$. Adding this class to $B_{i}$, we contradict the maximality of $B_{i}$.

$\square$ (claim)

Let $B_{i}^{\prime} \subset B_{i}$ be such that $\hat{N}_{i} \cup B_{i}^{\prime}$ is algebraically closed and $\left|B_{i}^{\prime}\right|=F(i)$. Let $N_{i} \prec M$ be prime over $\hat{N}_{i} \cup B_{i}^{\prime}$. Clearly, $A \subset N_{i}$ and $\left|\psi_{i}\left(N_{i}\right)\right|=\left|N_{i}\right|=F(i)$. Further, by lemma $1, \psi_{j}\left(N_{i}\right) \subset \hat{N}_{i}$ for every $j<i$. Hence, by the construction of $\hat{N}_{i}, \psi_{j}\left(N_{i}\right)=\psi_{j}\left(N_{j}\right)$ as desired.

\section{References}

[1] John Addison, Leon Henkin, and Alfred Tarski, editors. The Theory of Models, Amsterdam, 1965. 1963 International Symposium at Berkeley, North-Holland.

[2] Timothy Bays. Multi-Cardinal Phenomena in Stable Theories. PhD thesis, University of California, Los Angeles, 1994.

[3] Julia Knight, Anand Pillay, and Charles Steinhorn. Definable sets in ordered structures II. Transactions of the American Mathematical Society, 295:593-605, 1986.

[4] Alistair Lachlan. A property of stable theories. Fundamenta Mathematica, 77:9-20, 1972.

[5] Anand Pillay and Charles Steinhorn. Definable sets in ordered structures I. Transactions of the American Mathematical Society, 295:565-92, 1986.

[6] Saharon Shelah. Categoricity of Classes of Models. PhD thesis, The Hebrew University, 1976.

[7] Richard Vaught. A Löwenheim-Skolem theorem for cardinals far apart. In Addison et al. [1], pages 390-401. 